\title{
Review Article \\ The Endocannabinoid System as a Therapeutic Target in Glaucoma
}

\author{
Elizabeth A. Cairns, ${ }^{1}$ William H. Baldridge, ${ }^{2,3}$ and Melanie E. M. Kelly ${ }^{1,3}$ \\ ${ }^{1}$ Department of Pharmacology, Dalhousie University, Halifax, NS, Canada B3H 4R2 \\ ${ }^{2}$ Department of Medical Neuroscience, Dalhousie University, Halifax, NS, Canada B3H 4R2 \\ ${ }^{3}$ Department of Ophthalmology and Visual Sciences, Dalhousie University, Halifax, NS, Canada B3H 4R2
}

Correspondence should be addressed to Melanie E. M. Kelly; melanie.kelly@dal.ca

Received 10 July 2015; Accepted 10 November 2015

Academic Editor: Maurice Ptito

Copyright ( 2016 Elizabeth A. Cairns et al. This is an open access article distributed under the Creative Commons Attribution License, which permits unrestricted use, distribution, and reproduction in any medium, provided the original work is properly cited.

\begin{abstract}
Glaucoma is an irreversible blinding eye disease which produces progressive retinal ganglion cell (RGC) loss. Intraocular pressure (IOP) is currently the only modifiable risk factor, and lowering IOP results in reduced risk of progression of the disorder. The endocannabinoid system (ECS) has attracted considerable attention as a potential target for the treatment of glaucoma, largely due to the observed IOP lowering effects seen after administration of exogenous cannabinoids. However, recent evidence has suggested that modulation of the ECS may also be neuroprotective. This paper will review the use of cannabinoids in glaucoma, presenting pertinent information regarding the pathophysiology of glaucoma and how alterations in cannabinoid signalling may contribute to glaucoma pathology. Additionally, the mechanisms and potential for the use of cannabinoids and other novel agents that target the endocannabinoid system in the treatment of glaucoma will be discussed.
\end{abstract}

\section{Introduction}

The endocannabinoid system (ECS) including cannabinoid receptors, cognate biosynthetic and degradative enzymes, and endocannabinoids, such as anandamide (AEA) and 2arachidonoylglycerol (2-AG), is present in both anterior and posterior ocular tissues including the retina (reviewed in [1]). The presence of these components supports an important role for the ocular ECS in the endogenous signalling of both the anterior and posterior eye. Consistent with this, application of cannabinoids to the eye produces a variety of effects, notably hyperemia, reduced tear production, and a reduction in intraocular pressure (IOP) [2] (reviewed in [1,3]). Of these effects, the IOP lowering properties of cannabinoids have attracted considerable attention with respect to the possibility of developing cannabinoid-based therapeutics for glaucoma $[1,3-6]$, a progressive irreversible blinding eye disease, which is the second leading cause of blindness worldwide [7].

Glaucoma represents a group of optic neuropathies characterized by cupping of the optic nerve head and selective retinal ganglion cell (RGC) loss. While IOP is a major modifiable risk factor, the exact relation between IOP and RGC death is not completely clear. Patients may have glaucoma without having elevated IOP or conversely have elevated IOP but not have glaucoma [7, 8]. However, regardless of the initial IOP, for every $\mathrm{mmHg}$ reduction in IOP, there is a $10 \%$ reduced risk of progression of the disorder [9]. While cannabinoids were initially exploited in glaucoma solely based on their IOP lowering properties [2], recent evidence suggests that modulation of the ECS may also be neuroprotective (reviewed in [1]).

This review will discuss the use of cannabinoids in glaucoma, presenting pertinent information regarding the pathophysiology of glaucoma and how alterations in cannabinoid signalling may contribute to glaucoma pathology. Additionally, the mechanisms and potential of cannabinoids as ocular hypotensive agents and neuroprotectants in the treatment of glaucoma will be discussed.

\section{The Endocannabinoid System in the Eye}

The endocannabinoid system is present throughout most ocular tissues, including anterior eye tissues responsible for 


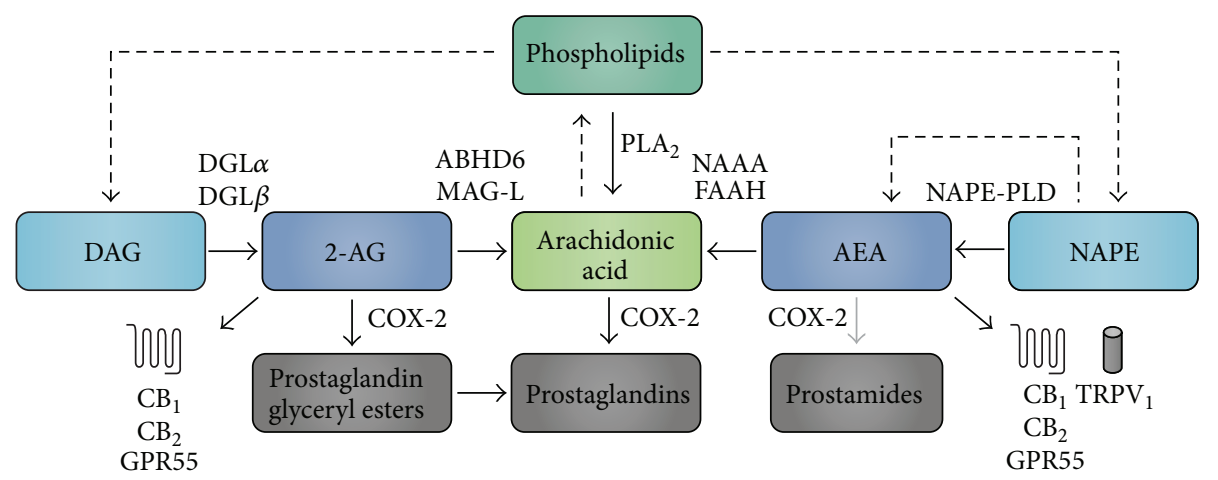

Figure 1: Overview of AEA and 2-AG production and metabolism. The endocannabinoids 2-AG and AEA are formed from arachidonic acid-containing phospholipids. 2-AG is formed from DAG by DGL $\alpha$ or DGL $\beta$ and is metabolized either via COX-2 to form prostaglandin glyceryl esters or by ABHD6 or MAG-L to form arachidonic acid. The production of AEA occurs through conversion of NAPE by either a NAPE-PLD dependent or independent pathway. Once formed, AEA is broken down by either NAAA or FAAH to form arachidonic acid or occasionally by COX-2 to form prostamides. Arachidonic acid can be synthesized via phospholipase $\mathrm{A}_{2}\left(\mathrm{PLA}_{2}\right)$ from phospholipids and is also broken down by COX-2, forming prostaglandins and other eicosanoids. Additionally, arachidonic acid can be converted back to a phospholipid [30, 31]. Dashed lines indicate multistep pathway; gray lines indicate weak pathway.

the generation of IOP (as outlined below), and in the retina (reviewed in [1]). The endocannabinoids 2-AG and AEA are found throughout the eye, with the exception of the lens [5, 10]. 2-AG and AEA both bind to cannabinoid 1 receptor $\left(\mathrm{CB}_{1}\right)$ and cannabinoid 2 receptor $\left(\mathrm{CB}_{2}\right) \cdot \mathrm{CB}_{1}$ is expressed in the ciliary body, trabecular meshwork, Schlemm's canal, and retina [11-19]. With respect to $\mathrm{CB}_{2}$, Cécyre and colleagues [20] reported that electroretinographic responses were altered in $\mathrm{CB}_{2}$ knockout mice, indicating that $\mathrm{CB}_{2}$ is present in the retina and may contribute to normal visual function. However, the localization of $\mathrm{CB}_{2}$ expression has been quite controversial. $\mathrm{CB}_{2} \mathrm{mRNA}$ has been reported in the retina [16], but the lack of good immunohistochemical markers has hampered studies attempting to study the expression pattern of the receptor. In 2011, López and colleagues [19] reported immunoreactive staining in the retinal pigmented epithelium and much of the inner retina; however, a recent study in a small number of nonhuman primates found that $\mathrm{CB}_{2}$ immunoreactivity was localized only to Müller cells [21] (also reported in in vitro data from primary cultures and retinal explants [22]). Additionally, pharmacological studies have suggested that $\mathrm{CB}_{2}$ may also be expressed in the anterior eye [23].

Several noncannabinoid receptor targets of endocannabinoids have also been localized to the eye. Transient receptor potential type vanilloid 1 receptor (TRPV1), a target of AEA, is expressed in the retina, including RGCs and retinal microglia [24]. GPR18 is a cannabinoid-related receptor that is activated by $\mathrm{N}$-arachidonoyl glycine (NAGly), a metabolite of AEA [25]. GPR18 has been localized to the cornea, ciliary epithelium, iris, and retina $[26,27]$. Investigation of the expression patterns of other cannabinoid-related receptors, such as GPR55 and GPR119, has been limited to date, although there is some evidence which supports the expression of GPR55 in the anterior eye and in rods in the retina $[28,29]$.

Endocannabinoid signalling is determined by the balance between production and degradation (Figure 1). Endocannabinoids are derived on demand from arachidonic acid-containing phospholipids after hydrolysis by phospholipases [30, 31]. Studies localizing the enzymes catalyzing the production of AEA and 2-AG in the eye have been minimal to date. However, two enzymes involved in the synthesis of 2-AG, diacylglycerol lipase- $\alpha$ and lipase- $\beta$ (DGL $\alpha$ and DGL $\beta$ ), have been localized to ocular structures in mouse [32]. As additional antibodies become available, it is more than likely that other enzymes will be localized to these tissues as well. AEA is primarily metabolized by the enzyme fatty acid amide hydrolase (FAAH), and also by $\mathrm{N}$-acylethanolamine-hydrolyzing acid amidase (NAAA) [30, 31, 33]. 2-AG is metabolized by both monoacylglycerol lipase (MAG-L) and $\alpha, \beta$-hydrolase domain-containing 6 (ABHD6) [31,34-36]. In addition to metabolizing arachidonic acid, evidence suggests that cyclooxygenase-2 (COX-2) can also directly metabolize both AEA and 2-AG to prostaglandinethanolamides (prostamides) and prostaglandin glyceryl esters, respectively [37, 38]. In fact, the affinity for and efficacy of the metabolism of 2-AG by COX-2 are comparable to those of arachidonic acid [39]. The nonselectivity of COX-2 between arachidonic acid and 2-AG highlights the interconnectedness of the ECS and the eicosanoid system [38]. FAAH and COX-2 immunoreactivity was found in the ciliary body, and in some retinal cells, including RGCs $[15,32,40,41]$. A similar pattern of immunoreactivity has been found for MAG-L and ABHD6 in the mouse retina [32]; however, so far only one study has reported the presence of MAG-L in porcine trabecular meshwork tissues, though this has yet to be confirmed with immunostaining [41]. NAAA was localized only to mouse retinal pigment epithelium and has yet to be investigated in the anterior eye [32].

\section{Alterations in ECS Signalling in Glaucoma and Retinal Disease}

Several studies have demonstrated fluctuations in endocannabinoid tone during disease states (reviewed in [42]). 
Deviations from homeostasis, including injury, inflammation, or even acute changes, will usually result in the elevation of at least one endocannabinoid in the tissues involved. This change may serve to help reestablish normal physiologic levels of other endogenous mediators and activate pathways to help protect the cells from death (reviewed in $[42,43]$ ). Nevertheless, chronic perturbation of the endocannabinoid system is not always protective and may under certain circumstances contribute to the pathology. Various disease states involve either up- or downregulation of endogenous cannabinoids, including many neurological disorders such as Parkinson's disease (increased AEA and decreased 2AG), Alzheimer's disease (increased 2-AG), amyotrophic lateral sclerosis (increased AEA and 2-AG), multiple sclerosis (decreased AEA and 2-AG), and neuropathic pain (increased AEA and 2-AG) (reviewed in [42, 43]).

Investigation of pathology-induced changes in the endocannabinoid system in the eyes of both human and animal models has so far been limited to relatively few studies (for review, see [1]). In diabetic retinopathy, 2-AG was increased in the iris, and AEA increased in the retina, ciliary body, and cornea. Similarly, AEA was increased in age-related macular degeneration in the retina, choroid, ciliary body, and cornea [10]. To date, only one study has looked at endocannabinoid levels in human glaucomatous eyes. Chen and colleagues [5] found that 2-AG and PEA ( $N$-palmitylethanolamide, an AEA analogue) were reduced in ciliary body, and PEA was also reduced in the choroid in postmortem eyes from glaucoma patients. Interestingly, there was no significant difference in AEA levels in any of the ocular tissues measured [5]; however, retinal AEA was found to be decreased 6 hours after reperfusion in a rat model of transient high IOP-induced ischemia, and this decrease in AEA was associated with increased FAAH activity [44].

Maihöfner and colleagues [40] found that COX-2 expression was significantly reduced in nonpigmented ciliary epithelial cells of human eyes with primary open-angle glaucoma. Furthermore, decreased endocannabinoid metabolites were found in sampled aqueous humor in these eyes [40]. This suggests that, under certain glaucomatous conditions, endocannabinoid metabolism might shift towards non-COX2-dependent mechanisms, perhaps a reflection of increased FAAH activity as suggested by Nucci et al. [44]. However, in a model of transient retinal ischemia in rats, COX-2 expression was found to be increased in the retina and was associated with neurodegeneration [45]. Further work is warranted in order to clarify the relationship between COX-2 and endocannabinoid signalling in glaucoma pathology.

A study by Nucci and colleagues [44] also found that $\mathrm{CB}_{1}$ and TRPV1 expression was reduced in the retinas of a rat model of ocular hypertension. Data from another study were contradictory; in the DBA2J mouse model of glaucoma, TRPV1 immunoreactivity appeared to be increased, particularly in the inner retina. Though it is possible that this is a reflection of shift in the expression of TRPV1 from the soma to dendrites, these results may demonstrate that changes in expression of TRPV1 in the retina may be isolated to specific cell types, making changes difficult to detect when analyzing whole retinal expression [24].
Taken together, the data indicate that changes in the ECS occurred in ocular pathology including glaucoma, suggesting that it may be possible to improve glaucomatous outcomes through therapeutic modification of the endocannabinoid system. For example, this may include strategies directed at regulation of IOP and/or increased RGC survival by restoring endocannabinoids to their nonpathological levels or by directly activating cannabinoid receptors and downstream signalling molecules to compensate for these changes.

\section{Cannabinoid Modulation of IOP}

All current pharmacotherapies for glaucoma target IOP [4648]. Cannabinoids modulate IOP, a phenomenon repeatedly demonstrated in studies using administration of endogenous or exogenous cannabinoids in rodents, rabbits, and nonhuman primates, and this ocular hypotensive action of cannabinoids has been a focus of studies of the therapeutic use of cannabinoids in the management of glaucoma. A few small human studies have reported efficacy of the phytocannabinoid $\Delta^{9}$-tetrahydrocannabinol $\left(\Delta^{9}\right.$-THC) and the synthetic $\mathrm{CB}_{1}$ agonist WIN 55,212-2 in decreasing IOP [4951]. Merritt et al. [52] found that topical application of $\Delta^{9}$ THC significantly decreased IOP in patients with glaucoma and did not elicit psychotropic effects noted in previous studies using smoked marijuana [53]. Another small study showed successful IOP reduction with topical application of the $\mathrm{CB}_{1}$ agonist WIN 55,212-2 in patients with glaucoma who were resistant to conventional treatment [49]. In general, the hypotensive effects of cannabinoids on IOP are largely due to actions at $\mathrm{CB}_{1}[4,49,54-56]$. However, these actions on IOP may also involve $\mathrm{CB}_{1}$-independent effects $[4,26,49,54-$ 58].

IOP is determined by the rate of aqueous humor production versus outflow. Aqueous humor is a transparent fluid produced by the eye that helps the eye keep its shape (important for proper optics) and acts as a modified circulatory system, removing wastes from avascular areas. Aqueous humor is produced by secretion from the ciliary body, which is located just beneath the iris (Figure 2), and is covered by a bilayered epithelium. Transporters on the ciliary epithelium allow the selective energy-dependent passage of certain solutes from the extracellular ciliary stroma into the posterior chamber [59]. $\mathrm{CB}_{1}, \mathrm{GPR} 18$, and various endocannabinoids have been localized to these tissues and may serve to modify secretion $[5,11,13,14,17,26]$. Aqueous humor is filtered out of the eye through two different outflow pathways: the trabecular meshwork pathway and the uveoscleral pathway. The contribution of each of these pathways to the overall outflow is subject to variability between species (3-80\%, depending on species, with significant interspecies variability) (reviewed in [60]).

The trabecular meshwork is located in the angle between the iris and cornea (Figure 2). Circulating aqueous humor will flow from the trabecular meshwork before being filtered through Schlemm's canal and exiting into the episcleral veins. Flow through this pathway may be altered by modifying resistance through the trabecular meshwork, modulated by 


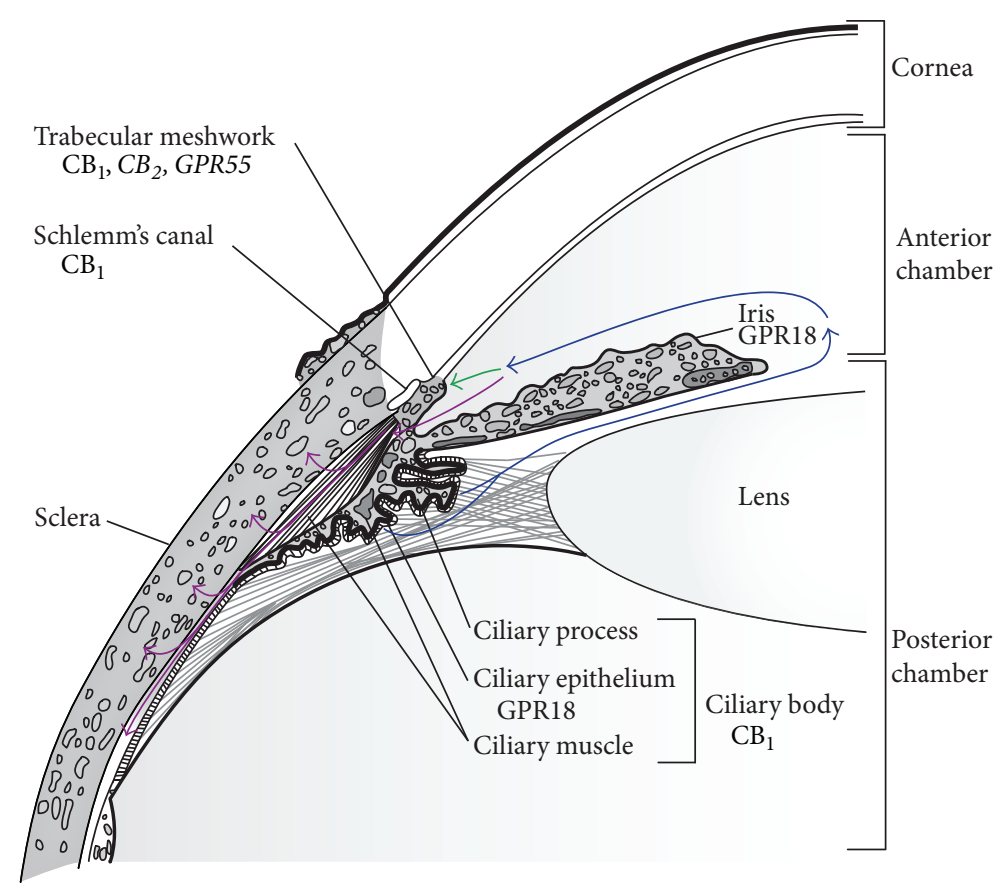

FIGURE 2: Cannabinoid-mediated alterations of the production and filtration of aqueous humor. Aqueous humor is formed by secretions from the ciliary body. Circulating aqueous humor (blue), flowing from the ciliary body in the posterior chamber to the anterior chamber, is filtered out of the eye through two different outflow pathways: the trabecular meshwork pathway (green) and the uveoscleral pathway (purple). The trabecular meshwork pathway involves the flow of aqueous humor through the trabecular meshwork to Schlemm's canal, where it will exit through the episcleral veins. The uveoscleral pathway involves the flow of aqueous humor from the iridocorneal angle to the posterior chamber through the ciliary body, and out through the supraciliary and suprachoroidal spaces. $\mathrm{CB}_{1}$, the major contributor to the IOP lowering effects of $\Delta^{9}$-THC and WIN 55,212-2, has been localized to the ciliary body, trabecular meshwork, and Schlemm's canal [11, 13-15, 17]. The IOP lowering effects of NAGly and Abn-CBD, and possibly CBG-DMH, are due to the activation of GPR18, which has been localized to the ciliary epithelium and iris $[26,58]$. Additional pharmacological evidence has suggested that $\mathrm{CB}_{2}$ and GPR55 are localized within the trabecular meshwork $[23,28]$; the contribution of these receptors to changes in IOP is unknown. COX-2 derived prostaglandins and prostamides are purported to exert actions through the uveoscleral pathway; however, the exact mechanism(s) is unclear [36, 62, 63]. Figure adapted from Riordan-Eva [94]. Italics indicate potential receptor localization which is not yet confirmed.

the nearby ciliary muscle and through the smooth-musclelike contractile properties of the trabecular meshwork itself [59]. $\mathrm{CB}_{1}, \mathrm{CB}_{2}$, and GPR55 receptors, as well as FAAH, have been localized at trabecular meshwork cells, indicating that they may also play a role in modulation at this site $[23,28,41$, $55,61]$.

The uveoscleral pathway involves the flow of aqueous humor from the iridocorneal angle to the posterior chamber through the ciliary body. From here, the aqueous enters the supraciliary and suprachoroidal spaces [60]. Analogues of the endocannabinoid-derived prostamides used to target this pathway include the prostamide $\mathrm{F}_{2 \alpha}$ analogue bimatoprost. The exact mechanism by which these drugs act is unknown; however, it is thought that this may involve binding to a novel heterodimerized prostaglandin receptor (FP/altFP receptor), causing downstream remodeling of the ciliary muscle, and enlargement of this outflow route $[36,62,63]$. Therefore, COX-2 metabolism of endocannabinoids and modulation of the ECS in the uveoscleral system may play an important role in the treatment of glaucoma.

Although $\Delta^{9}$-THC and WIN 55,212-2 are known to activate both $\mathrm{CB}_{1}$ and $\mathrm{CB}_{2}$, their ocular hypotensive properties are primarily due to $\mathrm{CB}_{1}$ activation and do not involve
$\mathrm{CB}_{2}[26,57,58]$. For example, in control rats, Szczesniak et al. [58] found that the $\mathrm{CB}_{2}$ antagonist AM630 did not reduce the IOP lowering effect of WIN 55,212-2, while blocking the $\mathrm{CB}_{1}$ receptor using the cannabinoid AM251 abolished this effect. This finding was confirmed using $\mathrm{CB}_{2}$ knockout mice, in which there was no significant difference in IOP reduction after application of WIN 55,212-2 compared to control. Interestingly, application of WIN 55,212-2 in $\mathrm{CB}_{1}$ knockout mice produced an increase in IOP via unidentified non- $\mathrm{CB}_{1}$ actions [57].

The ocular hypotensive effect of $\mathrm{CB}_{1}$ appears to be mediated, at least in part, by $\beta$-adrenergic receptors ( $\beta$ ARs) [57]. Both $\beta \mathrm{AR}$ agonists and antagonists reduce IOP and have been used to lower IOP in glaucomatous patients: $\beta \mathrm{AR}$ antagonists, such as timolol, reduce aqueous humor secretion, while $\beta$ AR agonists, such as isoproterenol, increase aqueous humor outflow by both trabecular and uveoscleral pathways. The $\mathrm{CB}_{1}$-mediated lowering of IOP is attenuated in $\beta A R$ knockout mice, as are $\beta$ AR agonist or antagonist effects in $\mathrm{CB}_{1}$ knockouts. Additionally, the catecholamine depleting agent reserpine was found to abolish the effects of both $\mathrm{CB}_{1}$ agonists and $\beta A R$ antagonists, suggesting that $C_{1}$ activation may inhibit the release of norepinephrine [57]. The lack of 
TABLE 1: Studies investigating cannabinoid-mediated neuroprotection in models of glaucoma.

\begin{tabular}{lcccl}
\hline Drug & Delivery & Study & Model & \multicolumn{1}{c}{$\begin{array}{l}\text { Neuroprotective effect versus vehicle } \\
\text { (treatment versus control) }\end{array}$} \\
\hline THC & IP & Crandall et al., 2007 [68] & Episcleral vein cauterization & $\sim 20-40 \%$ increase (10-20\% loss) \\
THC & IV & El-Remessy et al., 2003 [69] & Intravitreal NMDA & $\sim 9 \%$ of vehicle* \\
CBD & IV & El-Remessy et al., 2003 [69] & Intravitreal NMDA & $\sim 4 \%$ of vehicle* \\
WIN 55,212-2 & Topical & Pinar-Sueiro et al., 2013 [70] & Ischemia-reperfusion (high IOP) & $9.88 \%$ increase (2.45\% loss) \\
MetAEA & IVit & Nucci et al., 2007 [44] & Ischemia-reperfusion (high IOP) & $18.6 \%$ increase (9.4\% loss) \\
URB597 & IP & Nucci et al., 2007 [44] & Ischemia-reperfusion (high IOP) & $15.1 \%$ increase (12.9\% loss) \\
URB597 & IP & Slusar et al., 2013 [71] & Axotomy & 1 week, 19.5\% increase (27.9\% loss) \\
Celecoxib & IP & Sakai et al., 2009 [72] & Ischemia-reperfusion (high IOP) & $25.8 \%$ increase (39.1\% loss) \\
SC-58236 & IP & Ju et al., 2003 [45] & Ischemia-reperfusion (high IOP) & Central, 28.4\% increase (27.3\% loss) \\
& & & & Peripheral, 28\% increase (26.8\% loss) \\
\hline
\end{tabular}

IP, intraperitoneal; IV, intravenous; IVit, intravitreal; ${ }^{*}$ study reported quantification of tunnel positive cells only.

direct actions of $\beta \mathrm{AR}$ agonists in $\mathrm{CB}_{1}$ knockout mice was suggested to occur via desensitization of $\beta$ AR due to elevated noradrenergic tone. However, other interactions between $\mathrm{CB} 1$ and $\beta \mathrm{AR}$, such as heterodimerization, which may result in alterations in $\beta \mathrm{AR}$ expression and pharmacology, cannot be ruled out [57].

Other ECS modulators appear to have IOP lowering effects that are independent of $\mathrm{CB}_{1}$ or $\mathrm{CB}_{2}$. Two behaviourally inactive cannabinoids, abnormal cannabidiol (Abn-CBD) and cannabigerol-dimethyl heptyl (CBG-DMH), lowered IOP in normotensive rats, and these effects were not blocked by coapplication of either $\mathrm{CB}_{1}$ or $\mathrm{CB}_{2}$ antagonists. The ocular hypotensive effect of Abn-CBD and CBG-DMH was, however, abolished by O-1918, an antagonist at GPR18 [58]. NAGly, an agonist at GPR18 [64], was also reported to lower IOP. The ocular hypotensive action of NAGly was mediated independently of $\mathrm{CB}_{1}, \mathrm{CB}_{2}$, and GPR55, as demonstrated through the use of knockout mice [26]. Collectively, these data support a role for GPR18 in IOP regulation. While the actions of NAGly did not involve GPR55, PEA was reported to increase aqueous humor outflow through the trabecular meshwork via activation of GPR55 and peroxisome proliferator-activated receptor $\alpha$ [28].

Taken together, this work suggests that the ECS plays a prominent role in aqueous humor dynamics and that drugs targeting both $\mathrm{CB}_{1}$ and non- $\mathrm{CB}_{1} / \mathrm{CB}_{2}$ receptors may be useful as ocular hypotensives. An increasing understanding of the localization of components of the ECS to tissues involved in aqueous humor production and outflow and the function of the ECS in regulation of IOP under normal and pathological conditions is still required.

\section{Targeting the Endocannabinoid System for Novel Glaucoma Therapeutics}

Many patients, despite the use of IOP lowering drugs, continue to show progressive vision loss. This suggests that strategies which target IOP as well as providing neuroprotection may be beneficial [65]. There is no current approved neuroprotective drug for glaucoma, and this is most likely a reflection of the lack of knowledge of the mechanisms leading to glaucomatous RGC loss [65]. However, a few components of this pathway have been determined. RGCs ultimately die by apoptosis caused by activation of proapoptotic pathways, namely, caspase activation, by excessive intracellular calcium [66]. The source of this intracellular calcium has yet to be determined; however, this more than likely arises from multiple sources [66]. Once this late-phase apoptosis is activated, neuroprotection is no longer possible as cellular function is irreparably compromised [66, 67]. Therefore, therapies which may reduce the calcium load on the cell may provide novel strategies for the treatment of glaucoma.

Many studies examining ECS modulation in models of glaucoma have found that, aside from IOP lowering effects, modulating the ECS is neuroprotective; this includes use of ligands acting directly at cannabinoid receptors as well as modulators of cannabinoid metabolism (summarized in Table 1) [24, 44, 45, 68-74]. Additionally, the neuroprotective properties of these compounds have been demonstrated in pressure-independent models of RGC loss, including neural excitotoxicity and axotomy $[69,71,73]$, suggesting that ECS modulation may be neuroprotective in the retina independent of changes in IOP.

For example, Crandall and colleagues [68] found that, in a rat model of ocular hypertension, weekly administration of THC increased the number of surviving ganglion cells in both central and peripheral regions of affected retinas. Additionally, a recent study using topical administration of WIN 55,212-2 in a transient high IOP model indicated that significant cell loss was only seen in animals receiving vehicle and was not seen in cannabinoid-treated animals. This neuroprotective effect of WIN 55,212-2 was blocked with the $\mathrm{CB}_{1}$ antagonist AM251, and suggests that the increased RGC survival seen was due to the $\mathrm{CB}_{1}$ receptor activation [70].

Additional studies have confirmed these findings in other models. Hyperactivation of glutamate receptors has been used as a model of RGC death (with either NMDA or kainate), induced by intravitreal injection or direct bath application in vitro. Pretreatment with intravenous THC was 
neuroprotective in rat eyes injected with NMDA and was significantly, but not completely, attenuated with coadministration of the $\mathrm{CB}_{1}$ antagonist/inverse agonist SR141716A. These results suggested that $\mathrm{CB}_{1}$ is at least partially responsible for the neuroprotective effects seen with this model [69].

Endocannabinoid levels may fluctuate with disease. While decreased endocannabinoid tone has been associated with a number of pathologies, including glaucoma [5], increased endocannabinoid tone has been reported to be neuroprotective [42]. Therefore, strategies that reestablish or increase endocannabinoid levels may provide retinal neuroprotection. Studying the effects of AEA in vivo has been particularly difficult due to its instability; therefore, the majority of work that has been performed analyzing the role of AEA in neuroprotection has been either through the use of its stable analogue MetAEA or by decreasing AEA metabolism by inhibition of FAAH $[44,71]$. This latter strategy was effective in reducing RGC damage as a result of transient high IOP-induced ischemia whereby RGC loss was attenuated by administration of the FAAH inhibitor URB597. The neuroprotective actions of FAAH inhibition were reported to be due to actions at both $\mathrm{CB}_{1}$ and TRPV1 [44]. Interestingly, URB597 was also neuroprotective in a pressure-independent model of RGC loss (axotomy) [71], suggesting that these neuroprotective actions are at least partly independent of IOP modulation. Additionally, Slusar et al. [71] reported in this study that increased RGC survival after axotomy was associated with both $\mathrm{CB}_{1}$ - and $\mathrm{CB}_{2}$-dependent modulation of phagocytic microglia. In another study, MetAEA was also found to significantly recover Thy-1 expression in a model of ischemia-reperfusion. Like previous studies, this effect was blocked with the use of the $\mathrm{CB}_{1}$ antagonist SR141716, as well as the TRPV1 antagonist capsazepine [44].

While the role of 2-AG has not yet been extensively studied in models of glaucoma, several studies have found that 2-AG is neuroprotective in other models of neurodegenerative disorders (reviewed in [75]). The actions of 2AG have been examined in a model of inflammation using lipopolysaccharide (LPS), an inflammation-inducing component of Gram-negative bacteria, in Müller glial cultures. Here, 2-AG reduced proinflammatory cytokines and increased anti-inflammatory cytokines. These actions were thought to be mediated through both $\mathrm{CB}_{1}$ and $\mathrm{CB}_{2}$, as these effects were significantly diminished by antagonists acting at either of these receptors [22]. The finding that cannabinoids may modulate inflammatory mediators is significant given that several proinflammatory cytokines, such as tumor necrosis factor $\alpha(\mathrm{TNF} \alpha)$, have been reported to play a significant role in glaucomatous RGC death [76-78]. Therefore, reduction of proinflammatory cytokines may represent one mechanism of neuroprotection by cannabinoids.

In addition to reducing proinflammatory cytokines, there is evidence which supports the idea that $2-\mathrm{AG}$ may provide neuroprotection via a $\mathrm{CB}_{1}$-dependent reduction of $\mathrm{COX}$ 2 expression [79]. Increased COX-2 expression has been implicated as an important contributor to neuronal death in a variety of models, including NMDA-induced excitotoxicity and transient retinal ischemia [45, 72]. Reducing COX-2 upregulation was neuroprotective in these models; however, complete block of COX-2 expression in the eye may not be appropriate in the chronic treatment of glaucoma. In most tissues COX-2 is expressed only through induction; however, in the eye COX-2 is constitutively expressed in some amacrine and ganglion cells [45] as well as in the ciliary body [80], and therefore may have an important role in normal functioning. Additionally, while COX-2 may be upregulated in the retina, as mentioned previously, COX-2 expression was decreased in the ciliary body of human eyes with primary open-angle glaucoma and it is possible that further reduction in these tissues would not be beneficial [40]. Therefore, targeting upstream of COX-2, for example, by altering 2-AG rather than blocking COX-2 itself, may be more appropriate in order to reduce potential side effects.

\section{Use of Cannabinoids for Long-Term Treatment of Glaucoma}

The average glaucoma patient will require treatment over a number of decades; therefore, any drug designed to treat glaucoma will have to be both safe and effective when administered in the long term [6]. Despite the possibilities of formulating cannabinoids for localized ocular delivery, chronic use of conventional cannabinoid ligands poses inherent problems, notably, persistent receptor activation/blockage, tachyphylaxis, and the possibility of unwanted off-target actions and behavioral side effects [4]. Although inhalation of marijuana can lead to transient hyperemia and reduced tear production (reviewed in [1]), so far, most human studies using topical or oral cannabinoids have reported minimal issues $[49,50,52,81-83]$. One study reported 5 cases of attrition due to corneal irritation; however, 4 of these were given vehicle (mineral oil) [81]. Therefore, it would not appear that ECS-modulating drugs pose any additional considerations for topical delivery compared with current clinical topical treatments [46, 47].

To date, studies evaluating the effectiveness of cannabinoid ligands in long-term use have had variable results. Most studies have reported loss of effect of IOP lowering properties within a matter of hours $[49,50,57]$. However, a study by Hosseini and colleagues [84] found that chronic topical application of $0.5 \%$ WIN 55,212-2 was effective at reducing IOP for four weeks. This suggests that certain dosing parameters may allow for better long-term efficacy.

A number of alternative strategies may also aid in circumventing desensitization caused by exogenous cannabinoid administration and are showing significant promise in preclinical studies [71, 75, 85-87]. These include drugs that enhance endogenous endocannabinoid signalling. Endocannabinoids are produced locally on-demand in a stimulusdependent manner; therefore, modifying their metabolism offers the possibilities of improved target specificity. However, where there has been considerable success with the use of URB597 to increase AEA through FAAH inhibition, this does not necessarily seem to be the case for drugs that inhibit MAG-L. In a study of inflammatory pain, loss of effect through tolerance was an issue with chronic JZL184 administration; however, this was not an issue when the dose 
was lowered [86]. These studies suggest that increasing 2-AG levels may lead to greater cannabinoid receptor desensitization. Therefore, any drug regimen directed at increasing 2AG may require careful evaluation in order to identify an appropriate dose and dosing regimen to optimize therapeutic benefit while avoiding receptor desensitization.

Another approach to avoid loss of effect may be with the use of cannabinoid receptor allosteric modulators. Allosteric modulators bind to an alternative (allosteric) binding site distinct from the orthosteric site. Binding of the allosteric modulator induces conformational change in the receptor that affects the affinity and/or efficacy of the main orthosteric ligand (reviewed in [88]). Recently, an allosteric binding site was discovered on $\mathrm{CB}_{1}[89]$ and since then a few compounds have been synthesized and used to explore their modulatory potential [90-93]. $\mathrm{CB}_{1}$ positive allosteric modulators have the potential to stimulate endocannabinoid-mediated $\mathrm{CB}_{1}$ signalling in the absence of any significant $\mathrm{CB}_{1}$ desensitization. These agents also lack the behavioral side effects, and hence addictive potential, of cannabinoids that activate $\mathrm{CB}_{1}[90-$ 93], suggesting that they may be good candidate drugs to explore for the future treatment of glaucoma.

\section{Conclusion}

Increasing evidence suggests that modulation of the endocannabinoid system may show potential for the treatment of glaucoma. Administration of cannabinoids in experimental models can lower IOP and reduce RGC loss, possibly by independent mechanisms. Novel therapeutic strategies, including allosteric modulation and inhibition of endocannabinoid breakdown, may enhance the therapeutic effects seen with direct administration of cannabinoids. However, a better understanding of the components of the ECS, their tissuespecific expression, and the functional role of the ocular ECS is still lacking. This information remains essential in order to move forward with the identification of novel ECS drug targets to prevent retinal neuron loss.

\section{Conflict of Interests}

Melanie E. M. Kelly is a founding director of Panag Pharma Inc., a start-up company developing nonpsychotropic cannabinoids for pain and inflammation.

\section{Acknowledgment}

The writing of this review was supported by a grant from the Canadian Institutes of Health Research (CIHR) MOP-97768 (Melanie E. M. Kelly).

\section{References}

[1] S. Yazulla, "Endocannabinoids in the retina: from marijuana to neuroprotection," Progress in Retinal and Eye Research, vol. 27, no. 5, pp. 501-526, 2008.

[2] K. Green, "Marihuana in ophthalmology: past, present and future," Annals of Ophthalmology, vol. 11, no. 2, pp. 203-205, 1979.
[3] C. Nucci, M. Bari, A. Spanò et al., "Potential roles of (endo)cannabinoids in the treatment of glaucoma: from intraocular pressure control to neuroprotection," Progress in Brain Research, vol. 173, pp. 451-464, 2008.

[4] T. Järvinen, D. W. Pate, and K. Laine, "Cannabinoids in the treatment of glaucoma," Pharmacology \& Therapeutics, vol. 95, no. 2, pp. 203-220, 2002.

[5] J. Chen, I. Matias, T. Dinh et al., "Finding of endocannabinoids in human eye tissues: implications for glaucoma," Biochemical and Biophysical Research Communications, vol. 330, no. 4, pp. 1062-1067, 2005.

[6] A. J. Lee and I. Goldberg, "Emerging drugs for ocular hypertension," Expert Opinion on Emerging Drugs, vol. 16, no. 1, pp. 137-161, 2011.

[7] A. King, A. Azuara-Blanco, and A. Tuulonen, "Glaucoma," British Medical Journal, vol. 346, Article ID f3518, 9 pages, 2013.

[8] H. A. Quigley, "Glaucoma," The Lancet, vol. 377, no. 9774, pp. 1367-1377, 2011.

[9] A. Heijl, M. C. Leske, B. Bengtsson, L. Hyman, B. Bengtsson, and M. Hussein, "Reduction of intraocular pressure and glaucoma progression: results from the Early Manifest Glaucoma Trial," Archives of Ophthalmology, vol. 120, no. 10, pp. 1268-1279, 2002.

[10] I. Matias, J. W. Wang, A. S. Moriello, A. Nieves, D. F. Woodward, and V. Di Marzo, "Changes in endocannabinoid and palmitoylethanolamide levels in eye tissues of patients with diabetic retinopathy and age-related macular degeneration," Prostaglandins Leukotrienes and Essential Fatty Acids, vol. 75, no. 6, pp. 413-418, 2006.

[11] A. Porcella, P. Casellas, G. L. Gessa, and L. Pani, "Cannabinoid receptor $\mathrm{CB} 1 \mathrm{mRNA}$ is highly expressed in the rat ciliary body: implications for the antiglaucoma properties of marihuana," Molecular Brain Research, vol. 58, no. 1-2, pp. 240-245, 1998.

[12] T. Bisogno, I. Delton-Vandenbroucke, A. Milone, M. Lagarde, and V. Di Marzo, "Biosynthesis and inactivation of $\mathrm{N}$-arachidonoylethanolamine (anandamide) and $\mathrm{N}$ docosahexaenoylethanolamine in bovine retina," Archives of Biochemistry and Biophysics, vol. 370, no. 2, pp. 300-307, 1999.

[13] A. J. Straiker, G. Maguire, K. Mackie, and J. Lindsey, "Localization of cannabinoid CB1 receptors in the human anterior eye and retina," Investigative Ophthalmology \& Visual Science, vol. 40, no. 10, pp. 2442-2448, 1999.

[14] A. Straiker, N. Stella, D. Piomelli, K. Mackie, H. J. Karten, and G. Maguire, "Cannabinoid CB1 receptors and ligands in vertebrate retina: localization and function of an endogenous signaling system," Proceedings of the National Academy of Sciences of the United States of America, vol. 96, no. 25, pp. 14565-14570, 1999.

[15] S. Yazulla, K. M. Studholme, H. H. McIntosh, and D. G. Deutsch, "Immunocytochemical localization of cannabinoid CB1 receptor and fatty acid amide hydrolase in rat retina," The Journal of Comparative Neurology, vol. 415, no. 1, pp. 80-90, 1999.

[16] Q. Lu, A. Straiker, Q. Lu, and G. Maguire, "Expression of CB2 cannabinoid receptor mRNA in adult rat retina," Visual Neuroscience, vol. 17, no. 1, pp. 91-95, 2000.

[17] A. Porcella, C. Maxia, G. L. Gessa, and L. Pani, “The human eye expresses high levels of CB1 cannabinoid receptor mRNA and protein," The European Journal of Neuroscience, vol. 12, no. 3, pp. 1123-1127, 2000.

[18] W. D. Stamer, S. F. Golightly, Y. Hosohata et al., "Cannabinoid CB1 receptor expression, activation and detection of endogenous ligand in trabecular meshwork and ciliary process tissues," 
European Journal of Pharmacology, vol. 431, no. 3, pp. 277-286, 2001.

[19] E. M. López, P. Tagliaferro, E. S. Onaivi, and J. J. López-Costa, "Distribution of CB2 cannabinoid receptor in adult rat retina," Synapse, vol. 65, no. 5, pp. 388-392, 2011.

[20] B. Cécyre, N. Zabouri, F. Huppé-Gourgues, J.-F. Bouchard, and C. Casanova, "Roles of cannabinoid receptors type 1 and 2 on the retinal function of adult mice," Investigative Ophthalmology and Visual Science, vol. 54, no. 13, pp. 8079-8090, 2013.

[21] J. Bouskila, P. Javadi, C. Casanova, M. Ptito, and J.-F. Bouchard, "Müller cells express the cannabinoid CB2 receptor in the vervet monkey retina," The Journal of Comparative Neurology, vol. 521, no. 11, pp. 2399-2415, 2013.

[22] G. Krishnan and N. Chatterjee, "Endocannabinoids alleviate proinflammatory conditions by modulating innate immune response in muller glia during inflammation," Glia, vol. 60, no. 11, pp. 1629-1645, 2012.

[23] L. Zhong, L. Geng, Y. Njie, W. Feng, and Z.-H. Song, “CB2 cannabinoid receptors in trabecular meshwork cells mediate JWH015-induced enhancement of aqueous humor outflow facility," Investigative Ophthalmology \& Visual Science, vol. 46, no. 6, pp. 1988-1992, 2005.

[24] R. M. Sappington, T. Sidorova, D. J. Long, and D. J. Calkins, "TRPV1: contribution to retinal ganglion cell apoptosis and increased intracellular $\mathrm{Ca}^{2+}$ with exposure to hydrostatic pressure," Investigative Ophthalmology \& Visual Science, vol. 50, no. 2, pp. 717-728, 2009.

[25] M. Kohno, H. Hasegawa, A. Inoue et al., "Identification of $N$ arachidonylglycine as the endogenous ligand for orphan Gprotein-coupled receptor GPR18," Biochemical and Biophysical Research Communications, vol. 347, no. 3, pp. 827-832, 2006.

[26] M. D. Caldwell, S. S.-J. Hu, S. Viswanathan, H. Bradshaw, M. E. M. Kelly, and A. Straiker, "A GPR18-based signalling system regulates IOP in murine eye," British Journal of Pharmacology, vol. 169, no. 4, pp. 834-843, 2013.

[27] J. Macintyre, A. Dong, A. Straiker et al., "Cannabinoid and lipidmediated vasorelaxation in retinal microvasculature," European Journal of Pharmacology, vol. 735, no. 1, pp. 105-114, 2014.

[28] A. Kumar, Z. Qiao, P. Kumar, and Z.-H. Song, "Effects of palmitoylethanolamide on aqueous humor outflow," Investigative Ophthalmology \& Visual Science, vol. 53, no. 8, pp. 44164425, 2012.

[29] J. Bouskila, P. Javadi, C. Casanova, M. Ptito, and J.-F. Bouchard, "Rod photoreceptors express GPR55 in the adult vervet monkey retina," PLoS ONE, vol. 8, no. 11, Article ID e81080, 2013.

[30] V. Di Marzo, "Endocannabinoid signaling in the brain: biosynthetic mechanisms in the limelight," Nature Neuroscience, vol. 14, no. 1, pp. 9-15, 2011.

[31] D. Piomelli, "More surprises lying ahead. The endocannabinoids keep us guessing," Neuropharmacology B, vol. 76, pp. 228234, 2014.

[32] S. S.-J. Hu, A. Arnold, J. M. Hutchens et al., "Architecture of cannabinoid signaling in mouse retina," The Journal of Comparative Neurology, vol. 518, no. 18, pp. 3848-3866, 2010.

[33] K. Tsuboi, Y.-X. Sun, Y. Okamoto, N. Araki, T. Tonai, and N. Ueda, "Molecular characterization of $\mathrm{N}$-acylethanolaminehydrolyzing acid amidase, a novel member of the choloylglycine hydrolase family with structural and functional similarity to acid ceramidase," The Journal of Biological Chemistry, vol. 280, no. 12, pp. 11082-11092, 2005.
[34] M. Alhouayek, J. Masquelier, and G. G. Muccioli, "Controlling 2-arachidonoylglycerol metabolism as an anti-inflammatory strategy," Drug Discovery Today, vol. 19, no. 3, pp. 295-304, 2014.

[35] J. L. Blankman, G. M. Simon, and B. F. Cravatt, "A comprehensive profile of brain enzymes that hydrolyze the endocannabinoid 2-arachidonoylglycerol," Chemistry \& Biology, vol. 14, no. 12, pp. 1347-1356, 2007.

[36] D. F. Woodward, R. L. Jones, and S. Narumiya, "International union of basic and clinical pharmacology. LXXXIII: classification of prostanoid receptors, updating 15 years of progress," Pharmacological Reviews, vol. 63, no. 3, pp. 471-538, 2011.

[37] M. Alhouayek and G. G. Muccioli, "COX-2-derived endocannabinoid metabolites as novel inflammatory mediators," Trends in Pharmacological Sciences, vol. 35, no. 6, pp. 284-292, 2014.

[38] C. A. Rouzer and L. J. Marnett, "Endocannabinoid oxygenation by cyclooxygenases, lipoxygenases, and cytochromes P450: cross-talk between the eicosanoid and endocannabinoid signaling pathways," Chemical Reviews, vol. 111, no. 10, pp. 5899-5921, 2011.

[39] K. R. Kozak, S. W. Rowlinson, and L. J. Marnett, "Oxygenation of the endocannabinoid, 2-arachidonylglycerol, to glyceryl prostaglandins by cyclooxygenase-2," The Journal of Biological Chemistry, vol. 275, no. 43, pp. 33744-33749, 2000.

[40] C. Maihöfner, U. Schlötzer-Schrehardt, H. Gühring et al., "Expression of cyclooxygenase-1 and -2 in normal and glaucomatous human eyes," Investigative Ophthalmology \& Visual Science, vol. 42, no. 11, pp. 2616-2624, 2001.

[41] Y. F. Njie, Z. Qiao, Z. Xiao, W. Wang, and Z.-H. Song, "Narachidonylethanolamide-induced increase in aqueous humor outflow facility," Investigative Ophthalmology \& Visual Science, vol. 49, no. 10, pp. 4528-4534, 2008.

[42] V. Di Marzo and S. Petrosino, "Endocannabinoids and the regulation of their levels in health and disease," Current Opinion in Lipidology, vol. 18, no. 2, pp. 129-140, 2007.

[43] S. Giacoppo, G. Molino, M. Galuppo, and P. B. E. Mazzon, "Cannabinoids: new promising agents in the treatment of neurological diseases," Molecules, vol. 19, no. 11, pp. 18781-18816, 2014.

[44] C. Nucci, V. Gasperi, R. Tartaglione et al., "Involvement of the endocannabinoid system in retinal damage after high intraocular pressure-induced ischemia in rats," Investigative Ophthalmology \& Visual Science, vol. 48, no. 7, pp. 2997-3004, 2007.

[45] W.-K. Ju, K.-Y. Kim, and A. H. Neufeld, "Increased activity of cyclooxygenase-2 signals early neurodegenerative events in the rat retina following transient ischemia," Experimental Eye Research, vol. 77, no. 2, pp. 137-145, 2003.

[46] F. Carta, C. T. Supuran, and A. Scozzafava, "Novel therapies for glaucoma: a patent review 2007-2011," Expert Opinion on Therapeutic Patents, vol. 22, no. 1, pp. 79-88, 2012.

[47] P. L. Kaufman and C. A. Rasmussen, "Advances in glaucoma treatment and management: outflow drugs," Investigative Ophthalmology \& Visual Science, vol. 53, no. 5, pp. 2495-2500, 2012.

[48] K. Zhang, L. Zhang, and R. N. Weinreb, "Ophthalmic drug discovery: novel targets and mechanisms for retinal diseases and glaucoma," Nature Reviews Drug Discovery, vol. 11, no. 7, pp. 541-559, 2012.

[49] A. Porcella, C. Maxia, G. L. Gessa, and L. Pani, "The synthetic cannabinoid WIN55212-2 decreases the intraocular pressure in human glaucoma resistant to conventional therapies," The 
European Journal of Neuroscience, vol. 13, no. 2, pp. 409-412, 2001.

[50] I. Tomida, A. Azuara-Blanco, H. House, M. Flint, R. G. Pertwee, and P. J. Robson, "Effect of sublingual application of cannabinoids on intraocular pressure: a pilot study," Journal of Glaucoma, vol. 15, no. 5, pp. 349-353, 2006.

[51] P. Robson, "Therapeutic aspects of cannabis and cannabinoids," The British Journal of Psychiatry, vol. 178, pp. 107-115, 2001.

[52] J. C. Merritt, D. D. Perry, D. N. Russell, and B. F. Jones, "Topical delta 9-tetrahydrocannabinol and aqueous dynamics in glaucoma.," Journal of Clinical Pharmacology, vol. 21, no. 8-9, supplement, pp. 467S-471S, 1981.

[53] J. C. Merritt, W. J. Crawford, P. C. Alexander, A. L. Anduze, and S. S. Gelbart, "Effect of marihuana on intraocular and blood pressure in glaucoma," Ophthalmology, vol. 87, no. 3, pp. 222$228,1980$.

[54] F. Y. Chien, R.-F. Wang, T. W. Mittag, and S. M. Podos, "Effect of WIN 55212-2, a cannabinoid receptor agonist, on aqueous humor dynamics in monkeys," Archives of Ophthalmology, vol. 121, no. 1, pp. 87-90, 2003.

[55] Y. F. Njie, A. Kumar, Z. Qiao, L. Zhong, and Z.-H. Song, "Noladin ether acts on trabecular meshwork cannabinoid (CB1) receptors to enhance aqueous humor outflow facility," Investigative Ophthalmology \& Visual Science, vol. 47, no. 5, pp. 19992005, 2006.

[56] Z.-H. Song and C.-A. Slowey, "Involvement of cannabinoid receptors in the intraocular pressure-lowering effects of WIN55212-2," The Journal of Pharmacology and Experimental Therapeutics, vol. 292, no. 1, pp. 136-139, 2000.

[57] B. D. Hudson, M. Beazley, A.-M. Szczesniak, A. Straiker, and M. E. M. Kelly, "Indirect sympatholytic actions at $\beta$ adrenoceptors account for the ocular hypotensive actions of cannabinoid receptor agonists," The Journal of Pharmacology and Experimental Therapeutics, vol. 339, no. 3, pp. 757-767, 2011.

[58] A.-M. Szczesniak, Y. Maor, H. Robertson, O. Hung, and M. E. M. Kelly, "Nonpsychotropic cannabinoids, abnormal cannabidiol and canabigerol-dimethyl heptyl, act at novel cannabinoid receptors to reduce intraocular pressure," Journal of Ocular Pharmacology and Therapeutics, vol. 27, no. 5, pp. 427-435, 2011.

[59] M. M. Civan, "Formation of the aqueous humor: transport components and their integration," Current Topics in Membranes, vol. 62, pp. 1-45, 2008.

[60] M. P. Fautsch and D. H. Johnson, "Aqueous humor outflow: what do we know? Where will it lead us?" Investigative Ophthalmology \& Visual Science, vol. 47, no. 10, pp. 4181-4187, 2006.

[61] Y. F. Njie, F. He, Z. Qiao, and Z.-H. Song, "Aqueous humor outflow effects of 2-arachidonylglycerol," Experimental Eye Research, vol. 87, no. 2, pp. 106-114, 2008.

[62] Y. Liang, D. F. Woodward, V. M. Guzman et al., "Identification and pharmacological characterization of the prostaglandin FP receptor and FP receptor variant complexes," British Journal of Pharmacology, vol. 154, no. 5, pp. 1079-1093, 2008.

[63] S. D. Smid, "Role of prostaglandins and specific place in therapy of bimatoprost in the treatment of elevated intraocular pressure and ocular hypertension: a closer look at the agonist properties of bimatoprost and the prostamides," Clinical Ophthalmology, vol. 3, no. 1, pp. 663-670, 2009.

[64] D. McHugh, S. S. J. Hu, N. Rimmerman et al., " $N$-arachidonoyl glycine, an abundant endogenous lipid, potently drives directed cellular migration through GPR18, the putative abnormal cannabidiol receptor," BMC Neuroscience, vol. 11, article 44, 2010.
[65] E. R. Tamm, L. Schmetterer, and F. Grehn, "Status and perspectives of neuroprotective therapies in glaucoma: the European Glaucoma Society White Paper," Cell and Tissue Research, vol. 353, no. 2, pp. 347-354, 2013.

[66] J. Qu, D. Wang, and C. L. Grosskreutz, "Mechanisms of retinal ganglion cell injury and defense in glaucoma," Experimental Eye Research, vol. 91, no. 1, pp. 48-53, 2010.

[67] M. R. Hara and S. H. Snyder, "Cell signaling and neuronal death," Annual Review of Pharmacology and Toxicology, vol. 47, pp. 117-141, 2007.

[68] J. Crandall, S. Matragoon, Y. M. Khalifa et al., "Neuroprotective and intraocular pressure-lowering effects of (-)Delta9tetrahydrocannabinol in a rat model of glaucoma," Ophthalmic Research, vol. 39, no. 2, pp. 69-75, 2007.

[69] A. B. El-Remessy, I. E. Khalil, S. Matragoon et al., "Neuroprotective effect of (-)Delta9-tetrahydrocannabinol and cannabidiol in N-methyl-D-aspartate-induced retinal neurotoxicity: involvement of peroxynitrite," American Journal of Pathology, vol. 163, no. 5, pp. 1997-2008, 2003.

[70] S. Pinar-Sueiro, J. Á. Zorrilla Hurtado, P. Veiga-Crespo, S. C. Sharma, and E. Vecino, "Neuroprotective effects of topical CB1 agonist WIN 55212-2 on retinal ganglion cells after acute rise in intraocular pressure induced ischemia in rat," Experimental Eye Research, vol. 110, pp. 55-58, 2013.

[71] J. E. Slusar, E. A. Cairns, A.-M. Szczesniak, H. B. Bradshaw, A. Di Polo, and M. E. M. Kelly, "The fatty acid amide hydrolase inhibitor, URB597, promotes retinal ganglion cell neuroprotection in a rat model of optic nerve axotomy," Neuropharmacology C, vol. 72, pp. 116-125, 2013.

[72] Y. Sakai, T. Tanaka, M. Seki et al., "Cyclooxygenase-2 plays a critical role in retinal ganglion cell death after transient ischemia: real-time monitoring of RGC survival using Thy-1EGFP transgenic mice," Neuroscience Research, vol. 65, no. 4, pp. 319-325, 2009.

[73] A. Mori, T. Ishii, T. Kuroki et al., “The prostanoid $\mathrm{EP}_{2}$ receptor agonist ONO-AE1-259-01 protects against glutamate-induced neurotoxicity in rat retina," European Journal of Pharmacology, vol. 616, no. 1-3, pp. 64-67, 2009.

[74] N. N. Osborne, G.-Y. Li, D. Ji et al., "Expression of prostaglandin PGE2 receptors under conditions of aging and stress and the protective effect of the EP2 agonist butaprost on retinal ischemia," Investigative Ophthalmology \& Visual Science, vol. 50, no. 7, pp. 3238-3248, 2009.

[75] J. Y. Xu and C. Chen, "Endocannabinoids in synaptic plasticity and neuroprotection," The Neuroscientist, vol. 21, no. 2, pp. 152168,2015

[76] M. Roh, Y. Zhang, Y. Murakami et al., "Etanercept, a widely used inhibitor of tumor necrosis factor- $\alpha$ (TNF- $\alpha)$, prevents retinal ganglion cell loss in a rat model of glaucoma," PLoS ONE, vol. 7, no. 7, Article ID e40065, 2012.

[77] R. Vohra, J. C. Tsai, and M. Kolko, "The role of inflammation in the pathogenesis of glaucoma," Survey of Ophthalmology, vol. 58, no. 4, pp. 311-320, 2013.

[78] X. Yang, C. Luo, J. Cai et al., "Neurodegenerative and inflammatory pathway components linked to TNF-alpha/TNFR1 signaling in the glaucomatous human retina," Investigative Ophthalmology \& Visual Science, vol. 52, no. 11, pp. 8442-8454, 2011.

[79] J. Zhang and C. Chen, "Endocannabinoid 2-arachidonoylglycerol protects neurons by limiting COX-2 elevation," The Journal of Biological Chemistry, vol. 283, no. 33, pp. 22601-22611, 2008. 
[80] F. Drago, S. Valzelli, I. Emmi, A. Marino, C. C. Scalia, and V. Marino, "Latanoprost exerts neuroprotective activity in vitro and in vivo," Experimental Eye Research, vol. 72, no. 4, pp. 479486, 2001.

[81] W. M. Jay and K. Green, "Multiple-drop study of topically applied 1\% delta 9-tetrahydrocannabinol in human eyes," Archives of Ophthalmology, vol. 101, no. 4, pp. 591-593, 1983.

[82] N. Plange, K. O. Arend, M. Kaup et al., "Dronabinol and retinal hemodynamics in humans," American Journal of Ophthalmology, vol. 143, no. 1, pp. 173-174, 2007.

[83] C. Gagliano, E. Ortisi, L. Pulvirenti et al., "Ocular hypotensive effect of oral palmitoyl-ethanolamide: a clinical trial," Investigative Ophthalmology and Visual Science, vol. 52, no. 9, pp. 60966100, 2011.

[84] A. Hosseini, F. A. Lattanzio, P. B. Williams, D. Tibbs, S. S. Samudre, and R. C. Allen, "Chronic topical administration of WIN-55-212-2 maintains a reduction in IOP in a rat glaucoma model without adverse effects," Experimental Eye Research, vol. 82, no. 5, pp. 753-759, 2006.

[85] D. Fernández-Suárez, M. Celorrio, J. I. Riezu-Boj et al., “The monoacylglycerol lipase inhibitor JZL184 is neuroprotective and alters glial cell phenotype in the chronic MPTP mouse model," Neurobiology of Aging, vol. 35, no. 11, pp. 2603-2616, 2014.

[86] S. Ghosh, L. E. Wise, Y. Chen et al., "The monoacylglycerol lipase inhibitor JZL184 suppresses inflammatory pain in the mouse carrageenan model," Life Sciences, vol. 92, no. 8-9, pp. 498-505, 2013.

[87] P. S. Katz, J. K. Sulzer, R. A. Impastato, S. X. Teng, E. K. Rogers, and P. E. Molina, "Endocannabinoid degradation inhibition improves neurobehavioral function, blood-brain barrier integrity, and neuroinflammation following mild traumatic brain injur," Journal of Neurotrauma, vol. 32, no. 5, pp. 297-306, 2015.

[88] D. Wootten, A. Christopoulos, and P. M. Sexton, "Emerging paradigms in GPCR allostery: implications for drug discovery," Nature Reviews Drug Discovery, vol. 12, no. 8, pp. 630-644, 2013.

[89] M. R. Price, G. L. Baillie, A. Thomas et al., "Allosteric modulation of the cannabinoid CB1 receptor," Molecular Pharmacology, vol. 68, no. 5, pp. 1484-1495, 2005.

[90] R. G. Pertwee, "The pharmacology of cannabinoid receptors and their ligands: an overview," International Journal of Obesity, vol. 30, supplement 1, pp. S13-S18, 2006.

[91] R. A. Ross, "Tuning the endocannabinoid system: allosteric modulators of the $\mathrm{CB}_{1}$ receptor," British Journal of Pharmacology, vol. 152, no. 5, pp. 565-566, 2007.

[92] F. A. Pamplona, J. Ferreira, O. M. de Lima Jr. et al., "Antiinflammatory lipoxin A4 is an endogenous allosteric enhancer of CB1 cannabinoid receptor," Proceedings of the National Academy of Sciences of the United States of America, vol. 109, no. 51, pp. 21134-21139, 2012.

[93] G. L. Baillie, J. G. Horswill, S. Anavi-Goffer et al., " $\mathrm{CB}_{1}$ receptor allosteric modulators display both agonist and signaling pathway specificity," Molecular Pharmacology, vol. 83, no. 2, pp. 322338, 2013.

[94] P. Riordan-Eva, "Anatomy \& embryology of the eye," in Vaughan \& Asbury's General Ophthalmology, P. Riordan-Eva and E. T. Cunningham, Eds., The McGraw-Hill Companies, New York, NY, USA, 18th edition, 2011. 

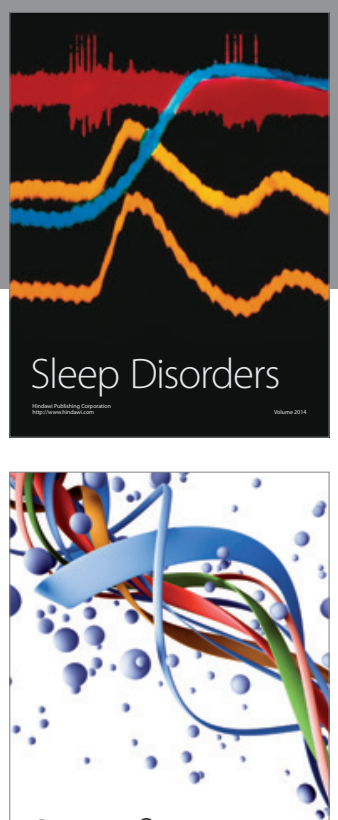

Scientifica
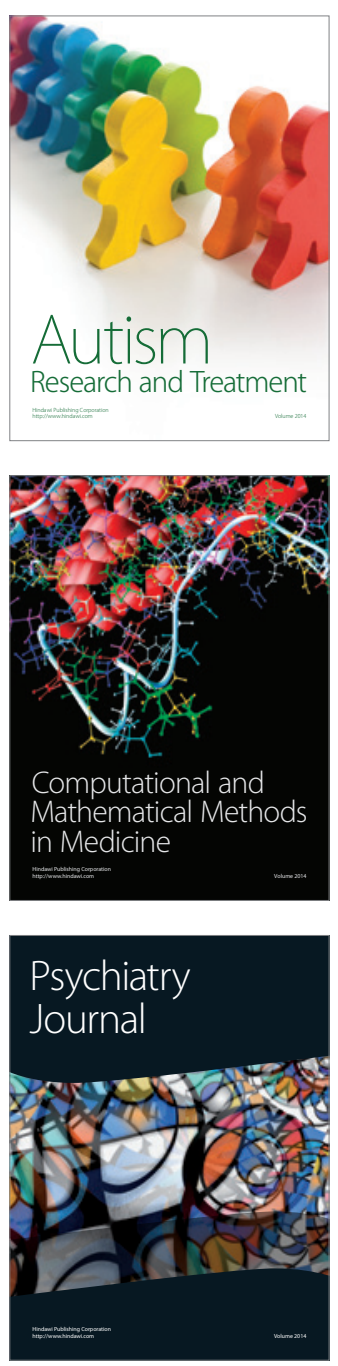
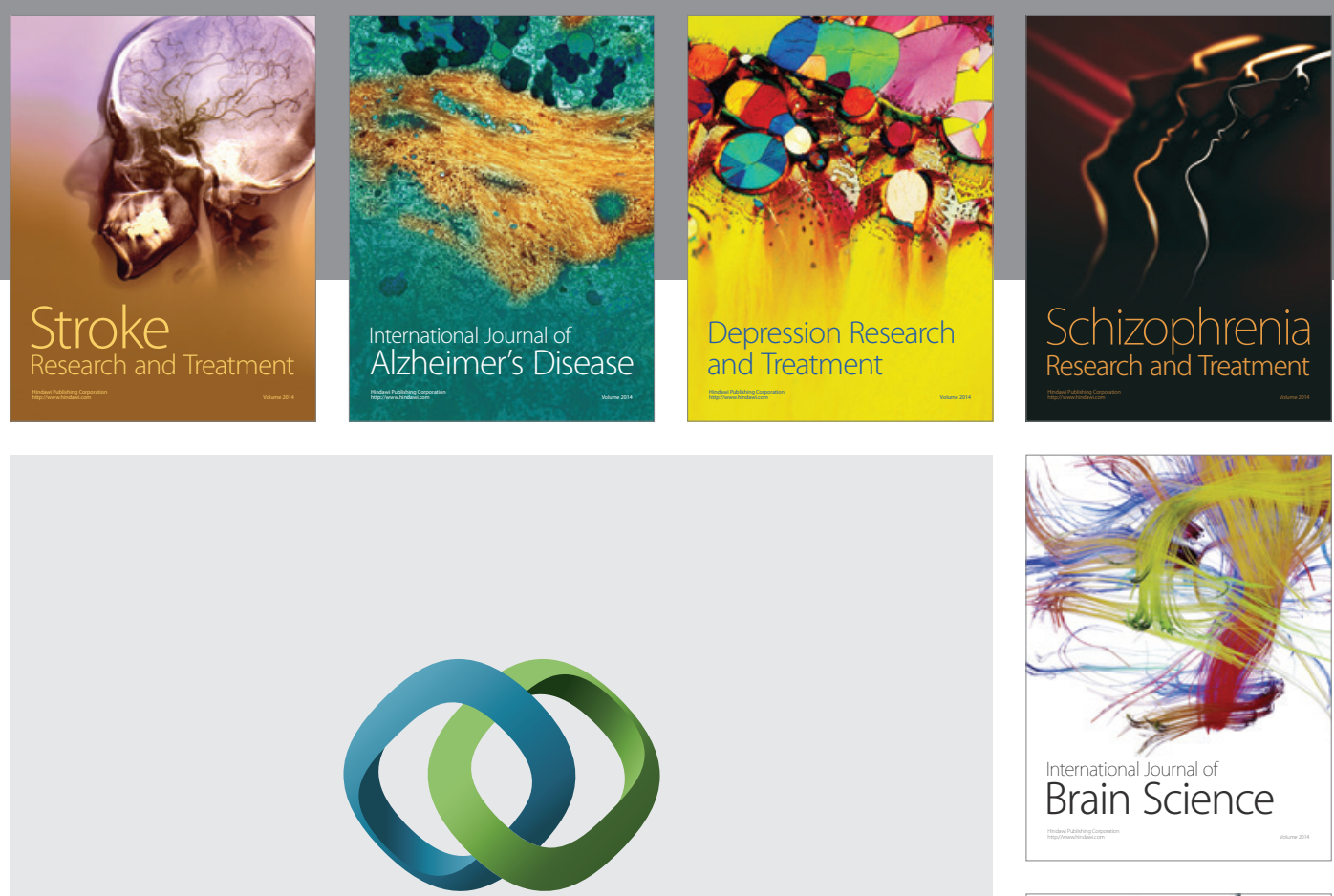

\section{Hindawi}

Submit your manuscripts at

http://www.hindawi.com
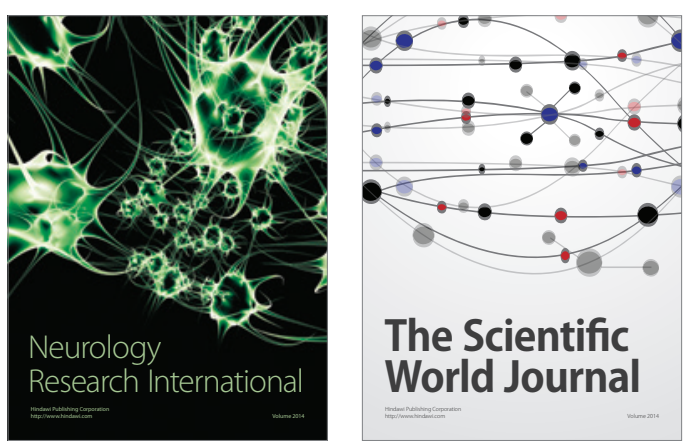

The Scientific World Journal

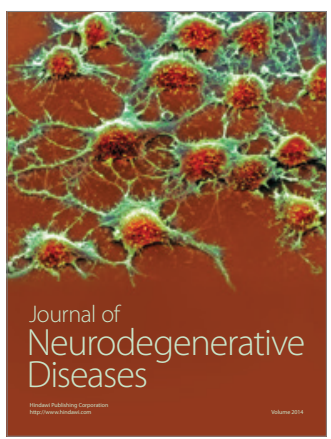

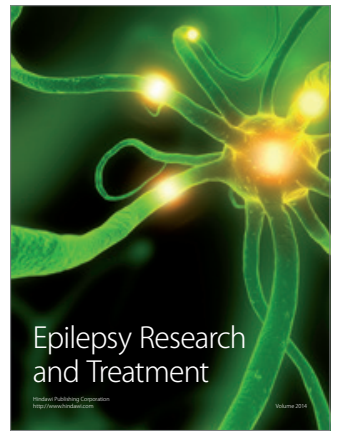

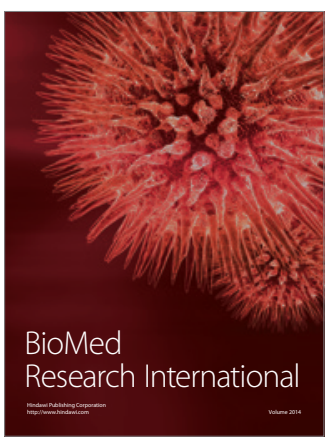

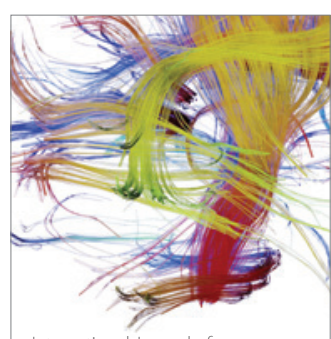

Brain Science

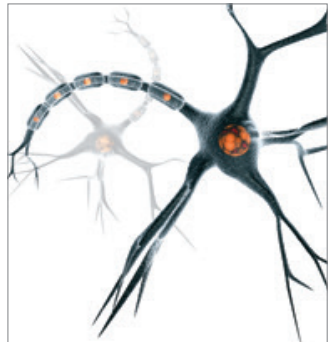

Neural Plasticity
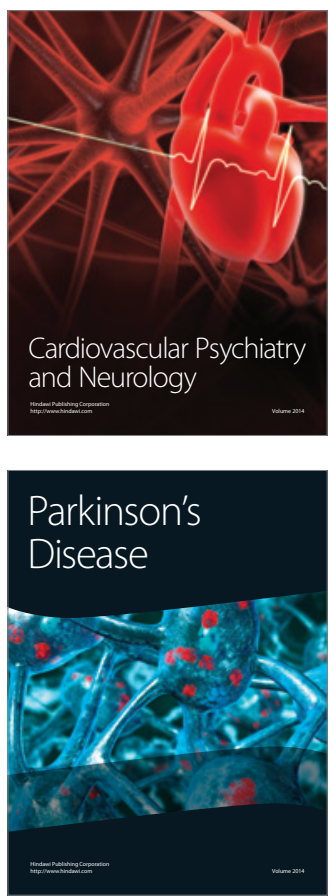